\title{
THE OPTIMUM PROCESSING OF CLIPPED SIGNALS: AN APPROACH BASED ON MINIMUM SIGNAL DISTORTION
}

\author{
R. G. KEATS and JOAN COOPER
}

(Received 18 September 1979)

(Revised 4 February 1980)

\begin{abstract}
The minimization of signal distortion was one approach applied successfully in the theory of optimum signal detection for arrays [3]. The processors considered operated on the input as received.

In some applications it is desirable to clip the received signal before processing and the problem of optimum processing of such clipped signals then arises. Several approaches to this problem are being studied, but the present paper is concerned with that based on minimum signal distortion.
\end{abstract}

\section{Introduction}

Criteria for the optimization of acoustic signal detection were discussed in detail by Edelblute, Fisk and Kinnison [3] with relation to an array processor in which the input from each of $K$ receivers in an array was linearly weighted before they were further processed. These authors showed that three approaches to the problem of optimum weightings gave precisely the same results at a single frequency. The three approaches studied by them were

(i) the maximization of array gain,

(ii) the minimization of signal distortion and

(iii) the Neyman-Pearson likelihood ratio.

This work combined the results of several earlier papers, including those by Bryn [1], Faran and Hills [6] and Mermoz [9].

For several years there has been considerable interest in signal processors which operate on clipped inputs, the input $I_{i}(t)$ from each receiver being first transformed to sgn $\left[I_{i}(t)\right]$ before any further processing is carried out. Such a processor has been 
described and its performance discussed by several authors (see, for example, $[2,4,5,7,11,12])$. However, there appears to be little or no published work on the problem of optimum weighting of clipped inputs. Clearly appropriate processing of the clipped signals should give improved performance over the simple sum and square processing discussed in $[7,11,12]$.

A recent paper by de Maré [8] describes the application of Wiener theory [13] to the reconstruction of a stationary Gaussian process from its sign changes. The success of this work suggests that the second approach of Edelblute et al. [3], namely the minimization of signal distortion, may prove useful in developing optimum processing methods for clipped signals. This suggestion is explored further in the sequel. However, it is most unlikely that the three different approaches used in [3] will yield identical results when applied to clipped data and other approaches to the problem including the remaining two from [3] are being explored.

The problem discussed in this paper is displayed in Figure 1. The inputs to each receiver, $R_{i}$, consist of a signal $S_{i}$ plus noise $N_{i}$. These inputs, which are assumed to be

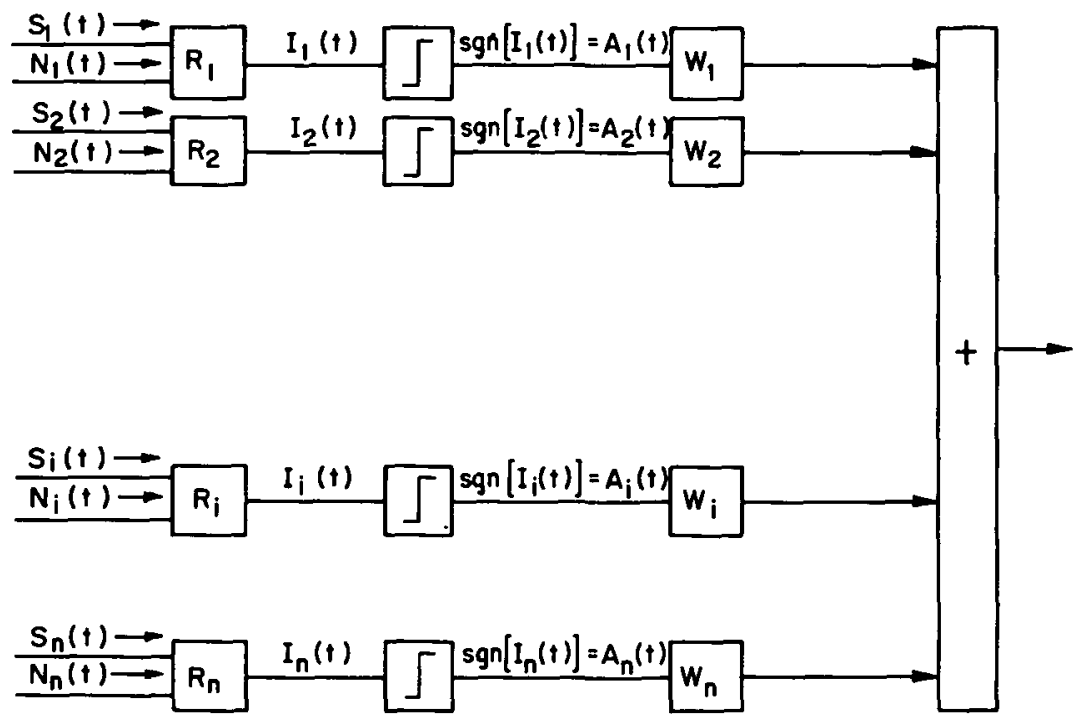

Fig. 1. A weighted clipped signal processor.

realizations of stationary normal random processes, are first clipped and then linearly filtered using weighting functions $W_{i}=W_{i}(u)$. It is proposed to use the criterion of minimum signal distortion in order to develop optimum weighting functions. 


\section{Notation and assumptions}

Throughout this paper the following notation and assumptions will apply; further assumptions may be introduced as they arise in particular cases.

\subsection{Input signal and noise}

The input signal at the $i$ th receiver, $i=1,2, \ldots, n$, is denoted by $S_{i}(t)$, which is a realization of a normal, stationary, ergodic, random process having zero mean and variance $\sigma^{2}{ }_{i}$. The signal to be detected will be represented by $S_{0}(t)$; it may equal one of the $S_{i}(t)$.

The noise at the $i$ th receiver is denoted by $N_{i}(t)$ which is also a realization of a normal, stationary, ergodic, random process having zero mean and variance $\sigma^{2}{ }_{i}$.

\subsection{Covariance and correlation functions}

The symbol $R$ with appropriate subscripts and superscripts is used to represent covariance functions; similarly, $\rho$ will represent a correlation function. Thus

$$
\begin{aligned}
R_{i j}^{S}(\tau) & =E\left[S_{i}(t) S_{j}(t+\tau)\right], \\
R_{i j}^{S N}(\tau) & =E\left[S_{i}(t) N_{j}(t+\tau)\right]
\end{aligned}
$$

and

$$
\sigma_{i}^{2} \rho_{i}^{S}(\tau)=E\left[S_{i}(t) S_{i}(t+\tau)\right]
$$

\subsection{Fourier transforms}

Script letters are used to denote spectral density functions, cross spectral densities and Fourier transforms in general. Thus

$$
\mathscr{R}_{i j}^{S}(\omega)=\int_{-\infty}^{\infty} R_{i j}^{S}(t) e^{-i \omega t} d t
$$

\subsection{Clipped output and other notation}

The clipped output from the $i$ th receiver is represented by $A_{i}(t)=\operatorname{sgn}\left[I_{i}(t)\right]$, where $I_{i}(t)=S_{i}(t)+N_{i}(t)$ is the input to that receiver.

Two additional symbols, $a_{i}$ and $c_{i}$, defined below, will also be used. Since we are dealing with a detection problem it will be assumed that $\sigma^{2}{ }_{i}^{S} \ll \sigma^{2 N}$ and, accordingly, $a_{i}$ and $\left|c_{i}\right|$ are both small. The definitions are

$$
a_{i}=\frac{\sigma_{0}^{S}}{\left(\sigma_{0}^{2 S}+\sigma_{i}^{2 N}\right)^{\frac{1}{2}}}
$$


and

$$
c_{i}=\left(\frac{2}{\pi}\right)^{\frac{1}{2}} \mathscr{S}_{0}^{t}(\omega) e^{i \omega t_{i}} a_{i}
$$

where

$$
\mathscr{S}_{0}(\omega)=\int_{-\infty}^{\infty} \rho_{0}^{S}(\tau) e^{-i \omega \tau} d \tau
$$

\section{The Wiener approach}

The second approach to their problem was also referred to by Edelblute et al. [3] as the Wiener approach. In his paper [8], de Mare investigated the problem of reconstructing a stationary normal process from its sign changes. He considered a process such as $S(t)$ above, with unit variance, and constructed a linear filter $W(u)$ such that

$$
E\left[S(t)-\int_{-\infty}^{\infty} \operatorname{sgn}\{S(t-u)\} W(u) d u\right]^{2}
$$

is a minimum. This is a classical Wiener problem involving a non causal filter. The examples given in [8] appear most encouraging, certainly sufficiently so to suggest a similar approach to the present problem. Accordingly, one seeks weighting functions $W_{i}(u)$ which minimize the quantity

$$
E\left[S_{0}(t)-\sum_{i=1}^{n} \int_{-\infty}^{\infty} A_{i}(t-u) W_{i}(u) d u\right]^{2} .
$$

It is well known [13] that the solution to this problem is contained in the $n$ equations

$$
E\left[\left\{S_{0}(t)-\hat{S}_{0}(t)\right\} \cdot\left\{A_{i}(t-u)\right\}\right]=0, \quad i=1, \ldots, n,
$$

where $S_{0}(t)$ is estimated by

$$
\hat{S}_{0}(t)=\sum_{i=1}^{n} \int_{-\infty}^{\infty} A_{i}(t-u) W_{i}(u) d u .
$$

Each of these $n$ equations can be written in the form

$$
\sum_{j=1}^{n} \int_{-\infty}^{\infty} R_{i j}^{A}(u-v) W_{j}(v) d v=R_{0 i}^{S A}(-u)
$$

where

$$
\begin{aligned}
R_{i j}^{A}(\tau) & =E\left[A_{i}(t) A_{j}(t+\tau)\right] \\
& =\frac{2}{\pi} \arcsin \rho_{i j}^{I}(\tau) \dagger,
\end{aligned}
$$

$\dagger$ This well-known result may be obtained as a simple application of Plackett [10]. 
with

and

$$
\rho_{i j}^{I}(\tau)=\frac{E\left[\left\{S_{i}(t)+N_{i}(t)\right\}\left\{S_{j}(t+\tau)+N_{j}(t+\tau)\right\}\right]}{\left(\sigma^{2}{ }_{i}{ }^{S}+\sigma^{2}{ }_{i}{ }^{\frac{1}{2}}\left(\sigma^{2}{ }^{2}{ }_{j}+\sigma^{2^{N}}\right)^{\frac{1}{2}}\right.}
$$

$$
\begin{aligned}
R_{0 i}^{S A}(\tau)= & E\left[S_{0}(t) A_{i}(t+\tau)\right] \\
& =\left(\frac{2}{\pi}\right)^{\frac{1}{2}} \frac{E\left[\left\{S_{0}(t)\right\}\left\{S_{i}(t+\tau)+N_{i}(t+\tau)\right\}\right]}{\left(\sigma^{2}{ }_{i}^{S}+\sigma^{2 N}{ }_{i}^{\frac{1}{2}}\right.} .
\end{aligned}
$$

This last result (4) can be derived by noting that

$$
\begin{aligned}
E\left[S_{0}(t) A_{i}(t+\tau)\right]= & E\left[S_{0}(t) \mid I_{i}(t+\tau) \geqslant 0\right]\left[P\left\{I_{i}(t+\tau) \geqslant 0\right\}\right] \\
& +E\left[-S_{0}(t) \mid I_{i}(t+\tau)<0\right]\left[P\left\{I_{i}(t+\tau)<0\right\}\right] \\
= & \frac{1}{2}\left\{E\left[S_{0}(t) \mid I_{i}(t+\tau) \geqslant 0\right]-E\left[S_{0}(t) \mid I_{i}(t+\tau)<0\right]\right\},
\end{aligned}
$$

which can be shown to equal

$$
2 \int_{0}^{\infty} d y \int_{-\infty}^{\infty} x f(x, y) d x
$$

where $f(x, y)$ is the joint probability density function of $S_{0}(t)$ and $I_{i}(t+\tau)$. Substituting for the bivariate normal density function $f(x, y)$ and performing the integrations gives

$$
E\left[S_{0}(t) A_{i}(t+\tau)\right]=\left(\frac{2}{\pi}\right)^{\frac{1}{t}} \frac{E\left[S_{0}(t) I_{i}(t+\tau)\right]}{\left(\sigma_{i}^{2 S}+\sigma_{i}^{2 N}\right)^{\frac{1}{2}}} .
$$

Taking Fourier transforms of both sides of (3), the $n$ equations become

$$
\sum_{j=1}^{n} \mathscr{R}_{i j}^{A}(\omega) \mathscr{W}_{j}(\omega)=\mathscr{R}_{0 i}^{S A^{*}}(\omega), \quad i=1, \ldots, n,
$$

where ${ }^{*}$ denotes complex conjugate, or in matrix form

$$
\mathbf{R} \mathbf{w}=\mathbf{r}^{*} .
$$

Assuming $\mathbf{R}^{-1}$ exists, the required vector $\mathbf{w}$ is given by

$$
\mathbf{w}=\mathbf{R}^{-1} \mathbf{r}^{*} \text {. }
$$

Theoretically, no further problems exist but, in general, the calculation of the several covariance functions and their transforms will be possible only by using numerical methods. However, various simplifications can be made in order to obtain some appreciation of this Wiener approach. Such simplifications are made in this section in order to carry the analysis further forward and also in the following section where some examples are given. 
We now make the following additional assumptions:

(a) $E\left[S_{i}(t) N_{j}(t+\tau)\right]=0$,

(b) $S_{0}(t)$ and $S_{i}(t)$ differ only by a time shift; thus

$$
\begin{aligned}
E\left[S_{0}(t) S_{i}(t+\tau)\right] & =E\left[S_{0}(t) S_{0}\left(t+\tau-t_{i}\right)\right] \\
& =\sigma_{0}^{2 S} \rho_{0}^{S}\left(\tau-t_{i}\right),
\end{aligned}
$$

and

(c) $E\left[N_{i}(t) N_{j}(t+\tau)\right]=0, i \neq j$.

The covariance functions then become

$$
R_{i i}^{A}(\tau)=\frac{2}{\pi} \arcsin \left[a_{i}^{2} \rho_{0}^{S}(\tau)+\left(1-a_{i}^{2}\right) \rho_{i}^{N}(\tau)\right],
$$

and

$$
R_{i j}^{A}(\tau)=\frac{2}{\pi} \arcsin \left[a_{i} a_{j} \rho_{0}^{S}\left(\tau-t_{j}+t_{i}\right)\right],
$$

the vector $\mathbf{r}^{*}$ of equation (6) has components

$$
r_{i}^{*}=\sigma_{0}^{S} \mathscr{S}_{0}^{\frac{1}{2}}(\omega)\left[\left(\frac{2}{\pi}\right)^{\frac{1}{t}} \mathscr{S}_{0}^{\frac{1}{2}}(\omega) e^{i \omega t_{1}} \frac{\sigma_{0}^{S}}{\left({\sigma^{2}}_{0}^{S}+{\sigma^{2}}_{i}^{N}\right)^{\frac{1}{t}}}\right]=\sigma_{0}^{S} \mathscr{S}_{0}^{\frac{1}{2}}(\omega) c_{i},
$$

the matrix $\mathbf{R}$ has diagonal terms

$$
\mathscr{R}_{i i}^{A}(\omega)=\int_{-\infty}^{\infty} \frac{2}{\pi} \arcsin \left[a_{i}^{2} \rho_{0}^{S}(\tau)+\left(1-a_{i}^{2}\right) \rho_{i}^{N}(\tau)\right] e^{-i \omega t} d \tau,
$$

and the off-diagonal terms of $\mathbf{R}$ are of the form

$$
\int_{-\infty}^{\infty} \frac{2}{\pi} \arcsin \left[a_{i} a_{j} \rho_{0}^{S}\left(\tau-t_{j}+t_{i}\right)\right] e^{-i \omega \tau} d \tau \text {. }
$$

Using the assumption in Section 2.4 that the $a_{i}$ are small, we have, approximately,

$$
\mathscr{R}_{i i}^{A}(\omega)=\int_{-\infty}^{\infty} \frac{2}{\pi} \arcsin \left[\rho_{i}^{N}(\tau)\right] e^{-i \omega \tau} d \tau
$$

and

$$
\mathscr{R}_{i j}^{A}(\omega)=\frac{2}{\pi} a_{i} a_{j} \mathscr{S}_{0}(\omega) e^{-i \omega\left(t_{j}-t i\right)}
$$

Equation (6) may then be written

$$
\mathbf{w}=\left[\mathbf{Q}+\mathbf{c c}^{H}\right]^{-1} \cdot \sigma_{0}^{S} \mathscr{S}_{\frac{1}{0}}^{\frac{1}{0}}(\omega) \mathbf{c},
$$

where $H$ denotes transpose conjugate. The matrix $\mathbf{Q}$ is diagonal with components $\mathscr{R}_{i i}^{A}(\omega)-\left|c_{i}\right|^{2} \simeq \mathscr{R}_{i i}^{A}(\omega)$, and the vector $\mathrm{c}$ has components $c_{i}$. 
Equation (10) is similar in form to equation (53) of [3] and accordingly the expression for $\mathbf{w}$ may be written

$$
\mathbf{w}=k \mathbf{Q}^{-1} \mathbf{c},
$$

where $k$ is a scalar which will in general depend on $\omega$. The differences between the components of $\mathbf{w}$ will arise from three factors:

(a) different noise variances at the input to each channel,

(b) different noise correlation functions, and

(c) phase differences between the input signals.

Factors (a) and (c) occur only in c while the factor (b) occurs only in $\mathbf{Q}^{-1}$. Since

$$
c_{i}=\left(\frac{2}{\pi}\right)^{\frac{1}{t}} \mathscr{S}_{0}^{t}(\omega) e^{i \omega t_{i}} \frac{\sigma_{0}^{S}}{\left(\sigma_{0}^{2}{ }_{0}^{S}+\sigma^{2^{N}}\right)^{\frac{1}{2}}} \text { and } \sigma_{0}^{2_{0}^{S}} \ll{\sigma^{2}}_{i}{ }^{N} \text {, }
$$

it is clear that the weighting function applied to each channel should include a term

$$
e^{i \omega t_{1}} / \sigma_{i}^{N}
$$

This is the only channel-dependent factor if $\rho_{i}^{N}(\tau)$ is independent of $i$; otherwise, the matrix $\mathbf{Q}$ must be inverted to determine the effects of the different correlation functions.

It is interesting to note that the denominator of (12) involves only the standard deviation of the noise in the $i$ th channel; a similar derivation for unclipped data would give a denominator involving the variance of the noise [3].

\section{Some examples with one or two receivers}

In this section four examples will be discussed involving one or two receivers. In comparing the performance of a clipped processor using weighting functions developed by the approach in this paper with that of a clipped processor using no weighting functions, the criterion used was the signal to noise ratio, SNR, defined as

$$
\mathrm{SNR}=\frac{E\left[\hat{S}_{0}(t)^{2}\right]_{\text {signal present }}-E\left[\hat{S}_{0}(t)^{2}\right]_{\text {signalabsent }},}{E\left[\hat{S}_{0}(t)^{2}\right]_{\text {signal absent }}},
$$

where $\hat{S}_{0}(t)$ is defined in equation (2), with $W_{i}(u)=\delta(u)$ for the case of no weighting.

Throughout this section it will be assumed that the signal and noise are uncorrelated and the noise processes from different channels are also uncorrelated.

Although it is clear that in the absence of weighting a clipped processor with one receiver would be useless as a signal detector, this is not generally the case when Wiener weighting is incorporated. To illustrate this fact two examples are given in 
Table 1. In the absence of weighting, $E\left[\hat{S}_{0}(t)^{2}\right]=1$ whether signal is present or absent; hence $S N R=0$. When Wiener weighting is used, the SNR is positive in both examples, although this effect is much more marked in the first than in the second.

TABLE 1

Signal to noise ratios with Wiener weighting and no weighting

\begin{tabular}{|c|c|c|c|c|c|}
\hline $\begin{array}{l}\text { No. of } \\
\text { receivers }\end{array}$ & $a_{i}^{2}=\frac{\sigma_{i}^{2 S}}{\sigma_{i}^{2^{S}}+\sigma_{i}^{2^{N}}}$ & $\begin{array}{l}\text { Correlation } \\
\text { of signal }\end{array}$ & $\begin{array}{l}\text { Correlation } \\
\text { of noise(s) }\end{array}$ & $\begin{array}{c}\text { SNR } \\
\text { no } \\
\text { weighting }\end{array}$ & $\begin{array}{c}\text { SNR } \\
\text { Wiener } \\
\text { weighting }\end{array}$ \\
\hline 1 & 0.1 & $\exp (-|\tau|)$ & $\exp (-2|\tau|)$ & 0 & 0.050 \\
\hline 1 & 0.1 & $\exp (-2|\tau|)$ & $\exp (-|\tau|)$ & 0 & 0.002 \\
\hline 2 & $\begin{array}{l}a_{1}^{2}=0.1 \\
a_{2}^{2}=0.05\end{array}$ & $\exp (-|\tau|)$ & $\begin{array}{l}\rho_{1}^{N}(\tau)=\exp (-2|\tau|) \\
\rho_{2}^{N}(\tau)=\exp (-3|\tau|)\end{array}$ & 0.05 & 0.138 \\
\hline 2 & $a_{1}^{2}=a_{2}^{2}=0.1$ & $\exp (-|\tau|)$ & $\rho_{1}^{N}(\tau)=\rho_{2}^{N}(\tau)=\exp (-|\tau|)$ & 0.063 & 0.087 \\
\hline
\end{tabular}

The table also contains the results for two examples involving two receivers; in both examples there are significant gains in signal to noise ratio when Wiener weighting is used.

The last example in the table corresponds to one of the earliest studies of processors with clipped inputs carried out by Faran and Hills [5]. The signal and both noise processes are assumed to have the same correlation function, in this case $\exp (-|\tau|)$; it is also assumed that $a_{1}=a_{2}=a$. After taking Fourier transforms of the Wiener equations, (3), the expression for each weighting function becomes

$$
\mathscr{W}_{1}(\omega)=\mathscr{W}_{2}(\omega)=\frac{\mathscr{R}_{01}^{S A}(\omega)}{\mathscr{R}_{12}^{A}(\omega)+\mathscr{R}_{11}^{A}(\omega)} .
$$

Some further manipulation yields $\mathscr{R}_{11}^{A}(\omega)$ as the real part of the expression

$$
\frac{4}{\pi}\left\{\frac{\pi}{2 i \omega}-\frac{\sqrt{ } \pi}{i \omega^{2}} \frac{\Gamma[(i \omega+1) / 2]}{\Gamma[(i \omega+2) / 2]}\right\}
$$

while

$$
\mathscr{R}_{01}^{S A}(\omega)=\left(\frac{2}{\pi}\right)^{\frac{1}{2}} \frac{2 \sigma^{S} a^{2}}{1+\omega^{2}}
$$

and, for $a^{2}$ small,

$$
\mathscr{R}_{12}^{A}(\omega)=\frac{4}{\pi}\left[\frac{a^{2}}{1+\omega^{2}}+\frac{a^{6}}{2\left(9+\omega^{2}\right)}+\frac{3 a^{10}}{2 \times 4\left(25+\omega^{2}\right)}+\ldots\right] .
$$

Thus the values of $\mathscr{W}_{1}(\omega)$, the transfer function corresponding to $W_{1}(u)$, are easily calculated. 
Using these weighting functions, the SNR value was found to be 0.087 for the case $a^{2}=0.1$, a significant increase over the value $2 a^{2} / \pi=0.064$ for SNR when no weighting is used. This result contrasts with the unclipped case, using the parameters of this example, where no increase in SNR can be obtained using Wiener or similar weighting [11].

\section{Discussion}

The work in this paper shows that, using a signal to noise ratio as criterion of performance, the Wiener approach to optimum weightings of the output of clipped receivers will produce significant improvements in the performance of a signal detection system over one in which no weightings are used. There is evidence based on an approximate analysis that such weightings will incorporate a factor to offset any time shift between channels and one which is inversely proportional to the standard deviation of the noise; the corresponding factor for the unclipped case is inversely proportional to the variance of the noise.

\section{Acknowledgements}

Part of this work was carried out under a grant to the University of Newcastle from the Department of Defence, Australia. It was completed while one of us (Keats) was visiting the University of Waterloo, Ontario, Canada.

\section{References}

[1] F. Bryn, "Optimum signal processing of three dimensional arrays, operating on Gaussian signals and noise", J. Acoust. Soc. Amer. 34 (1962), 282-297.

[2] M.C. Cheng, "The clipping loss in correlation detectors for arbitrary signal to noise ratios", I.E.E.E. Trans. on Information Theory IT-14 (1968), 382-389.

[3] D. J. Edelblute, J. M. Fisk and G. L. Kinnison, "Criteria for optimum-signal-detection theory for arrays", J. Acoust. Soc. Amer. 41 (1967), 199-205.

[4] H. Ekre, "Polarity coincidence correlation detection of a weak noise source", I.E.E.E. Trans. on Information Theory IT-9 (1963), 18-23.

[5] J. J. Faran and R. Hills Jr., "The application of correlation techniques to acoustic receiving systems", Technical Memo No. 28, Acoust. Res. Lab., Harvard (1952).

[6] J. J. Faran and R. Hills Jr., "Wide band directivity of receiving arrays", Technical Memo No. 31, Acoust. Res. Lab., Harvard (1953).

[7] R. G. Keats and V. K-K. Yu, "A generalization of the study of sum and square law signal processors with multiple clipped inputs", J. Austral. Math. Soc. B19 (1976), 294-315.

[8] J. de Maré, "Reconstruction of a stationary Gaussian process from its sign changes", J. Appl. Prob. 14 (1977), 38-57. 
[9] H. Mermoz, "Filtrage adapte et utilisation optimale d'une antenne", in Signal processing with emphasis on underwater acoustics (Proc. NATO Advan. Study Inst., 14-26 Sept. 1964), 163-294.

[10] R. L. Plackett, “A reduction formula for normal multivariate integrals", Biometrika 41 (1954), 351-360.

[11] P. M. Schultheiss and F. B. Tuteur, "Optimum and sub-optimum detection of directional Gaussian signals in an isotropic Gaussian noise field. Part II: Degradation of detectability due to clipping", I.E.E.E. Trans. on Military Electronics MIL-9 (1965), 208-211.

[12] J. Usher Jr., "Signal detection by arrays in noise fields with local variations", J. Acoust. Soc. Amer. 36 (1964), 1444-1449.

[13] N. Wiener, Extrapolation, interpolation and smoothing of stationary time series (M.I.T. Press and Wiley, New York, 1949).

\section{Department of Mathematics}

University of Newcastle

NSW 2308 